\title{
Civilisations
}

Revue internationale d'anthropologie et de sciences

humaines

66 | 2017

L'alcool rituel et les ethnographes

\section{"Drunk is beautiful"}

Boire au bord de l'eau : usage de l'alcool dans la communauté punk chinoise

\section{Nathanel Amar}

\section{OpenEdition}

\section{Journals}

Édition électronique

URL : http://journals.openedition.org/civilisations/4495

DOI : 10.4000/civilisations.4495

ISSN : 2032-0442

\section{Éditeur}

Institut de sociologie de l'Université Libre de Bruxelles

\section{Édition imprimée}

Date de publication : 31 août 2017

Pagination : 139-155

ISBN : 978-2-9602017-1-0

ISSN : 0009-8140

Référence électronique

Nathanel Amar, " "Drunk is beautiful" », Civilisations [En ligne], 66 | 2017, mis en ligne le 31 août 2020, consulté le 25 février 2021. URL : http://journals.openedition.org/civilisations/4495 ; DOI : https:// doi.org/10.4000/civilisations.4495 


\title{
«Drunk is beautiful »
}

\section{Boire au bord de l'eau : usage de l'alcool dans la communauté punk chinoise}

\author{
Nathanel AMAR
}

Résumé : . Cet article entend montrer en quoi la consommation d'alcool dans la communauté punk chinoise peut être rattachée à tout un répertoire de pratiques propres aux communautés déviantes $d u$ jianghu. Le jianghu, littéralement "rivières et lacs», représente depuis la dynastie des Zhou un espace physique et symbolique où les communautés marginales chinoises se retrouvent pour échapper au pouvoir central. Cette affinité entre punks et jianghu se retrouve dans les liens qui unissent les communautés de punks aux « mafias » locales. La consommation d'alcool sera ainsi analysée dans sa fonction rituelle et sociale, mais aussi politique, l'alcool s'opposant aux efforts de " civilisation » de la population par le gouvernement chinois, et permettant souvent de libérer la parole. L'alcool représente aussi un enjeu méthodologique pour le chercheur : comment se positionner soi-même face au risque de l'ivresse, alors que l'intégration au sein du terrain de recherche demande un engagement corporel total?

Mots-clé : Punk, sous-culture, Chine, observation participante, déviance.

\begin{abstract}
This paper tries to understand how alcohol consumption inside the Chinese punk community can be linked to a plurality of deviant practices specific to jianghu communities. Literally, "rivers and lakes", the concept of jianghu embodies, since the Zhou dynasty, a physical and symbolic space where Chinese maverick communities gathered to escape from the official authorities. This affinity between the punks and the jianghu can also be shown through the links between the punks and the local "mafias". Alcohol consumption can thus be analysed in its ritual and social function, but also through a political lens, since alcohol is opposed to the effort of the central government to "civilize" the population, and it often allows free speech. Alcohol consumption represents also a methodological issue for the researcher: how can the researcher deals with the risk of intoxication, when his integration to the fieldwork requires the total commitment of his body?
\end{abstract}

Keywords: Punk, subculture, China, participant observation, deviance. 
«We can live better without your socialism,

We can live better without the party,

We don't need more money, but only punk rock music,

We can live better with friends and beer!»

SMZB, For Friends and Beer.

\section{Introduction}

Fin mai 2013, le temps s'est réchauffé à Wuhan, réputée être l'une des trois « fournaises » de la Chine, avec les villes de Nankin et Chongqing. Le bar punk Wuhan Prison, tenu par Wu Wei, le chanteur du groupe mythique SMZB (生命之 饼), est rempli, comme tous les vendredis soirs. La foule s'est réfugiée à l'intérieur, profitant de la climatisation pour boire et écouter de la musique. A l'extérieur du bar, se sont attablées quatre personnes, qui jurent avec les clients habituels. La plupart torse nu, ce sont des hommes d'âge mûrs, autour de la cinquantaine, qui discutent avec un fort accent wuhanais. On peut reconnaître des personnalités du quartier où est situé le Wuhan Prison, à proximité d'une artère commerçante de Wuchang, le quartier des universités : ainsi, le patron du restaurant adjacent de "nouilles sèches et chaudes » (热干面), spécialité de Wuhan, ou encore un ancien policier du quartier, reconverti aujourd'hui « dans les affaires ». Wu Wei ouvre la porte du bar en portant une bouteille de whisky et six bières, qu'il pose sur la table. Les quatre hommes le remercient et continuent à discuter. Comme je l'apprendrai quelques minutes plus tard, ces quatre hommes ne sont pas des clients comme les autres, ils font partie de la « mafia », ou plutôt 《 société obscure 》 (littéralement 《黑社会 》) du quartier. A ce titre, dès qu'ils s'installent au bar, ils ont droit à de l'alcool gratuit en quantité, servi par le patron en personne. Fait unique dans le quartier, les punks du Wuhan Prison ne paient pas les services de « protection » de la mafia de Wuhan, comme tous les autres commerces du quartier, et plus spécifiquement les bars ${ }^{1}$, grâce aux bonnes relations qu'entretient $\mathrm{Wu}$ Wei avec les parrains locaux, ce qui l'oblige cependant à leur fournir gracieusement de l'alcool chaque fois qu'ils viennent dans son bar.

Cette proximité entre punk et « société obscure »n'est pourtant pas anodine. Elle trouve sa source dans les débuts du punk chinois, au milieu des années 1990, lorsque les groupes de punk peinaient à trouver un endroit où se produire. Ceux-ci durent alors négocier avec les propriétaires des bars à karaoké (KTV) des grandes villes, pour leur laisser louer des salles. Ces bars, hauts lieux de la prostitution, étaient bien évidemment tenus par des mafieux locaux, habitués à contourner les lois. Mais la relation des punks avec la « société obscure » chinoise est autrement plus complexe. Cette appartenance, revendiquée par bien des punks lors d'entretiens, n'implique pas seulement l'acceptation de hiérarchies implicites, comme l'indique la scène du Wuhan Prison, mais toute une organisation sociale alternative, faite de solidarités locales, de respect mutuel et de défiance envers l'autorité gouvernementale. Cette inscription dans une société alternative n'est pas propre aux punks de Wuhan, elle se retrouve dans bien d'autres composantes de l'underground chinois, que cela soit le rock (Grillot 2001), le punk ou le cinéma documentaire (Lü 2003). La notion d'underground, traduit

1 L'auteur, ayant ouvert un bar à Pékin, peut témoigner que ce phénomène est également présent dans la capitale. 
littéralement en chinois par dixia (地下), a longtemps symbolisé l'organisation des communautés contre-culturelles qui sont apparues en Chine dans les années 1990, tout en faisant référence à la culture non-officielle de la période maoïste nommée dixia wenxue (地下文学 - littérature clandestine), et notamment les romans recopiés à la main prêtés sous le manteau pendant la Révolution culturelle (Yang 2013). L'usage du terme dixia s'est depuis restreint, les cinéastes et rockers underground préférant aujourd'hui le terme de duli (独立), « indépendance » (Pickowicz et Zhang 2006) ou encore minjian (民间), populaire - au sens de non-officiel (Link et Madsen 2002), tandis que certains punks préfèrent définir leur style de vie comme «alternatif» (linglei - 另类), comme l'indique le témoignage de Mai Dian, ancien punk de Wuhan, sur son « éducation punk alternative » (Mai 2012). Plus encore que le dixia, l'ethos ${ }^{2}$ tout comme la sociabilité propre au mode de vie punk est parcouru par bien des aspects du jianghu (江湖), littéralement les « rivières et lacs », communauté de brigands et de hors-la-loi, popularisée par les romans d'aventure du XIV siècle, comme $A u$ bord de l'eau de Shi Nai'an. L'appartenance du punk au monde du jianghu ne fait pas de ses membres que de simples acteurs d'une contre-culture musicale, mais bien des participants d'une contre-société, possédant ses propres codes, lieux de sociabilités et rituels. À travers la dimension festive du punk, et en particulier la consommation d'alcool, se manifestent les spécificités propres au jianghu et ses pratiques. Cet article reviendra donc sur la signification du «jianghu », tant dans ses formes traditionnelles que contemporaines, avant d'analyser les usages sociaux de l'alcool au sein des communautés punks. Si certaines formes de sociabilités évoquées dans cet article se retrouvent dans d'autres communautés, nous entendons ici analyser les mécanismes spécifiques de la communauté punk à Wuhan, où j'ai effectué un terrain de recherche de 2012 à 2015, qui diffère d'autres communautés chinoises, comme nous le verrons. Enfin, nous reviendrons sur la position du chercheur durant l'enquête, en tentant de définir la relation éthylique qui unit le chercheur en sciences sociales et son sujet de recherche.

\section{La voie du jianghu : réactualisation des pratiques rituelles chez les punks chinois}

Il est compliqué de dater l'apparition du punk chinois, des débats sans fin entre les punks de la capitale et de Wuhan ne rendent pas la chronologie très lisible. Il est cependant admis que ce sont les disques dakou (打口) au milieu des années 1990 qui ont permis l'émergence de toute une scène musicale alternative. Le terme dakou - littéralement « cassé »- représente le surplus des disques qui étaient envoyés en Chine par les majors occidentaux pour être recyclés, et qui étaient cassés pour être rendus inutilisables. Mais un $\mathrm{CD}$ étant lu du centre du disque vers la périphérie, seules les dernières chansons étaient inécoutables (De Kloet 2010). Ces disques, peu onéreux, étaient vendus dans toutes les grandes villes chinoises et ont permis à toute une génération de jeunes chinois de découvrir des musiques auxquelles ils n'avaient pas accès. Reprenant le flambeau de la contestation musicale après le rock des années 1980, le punk s'est établi dans les grands centres urbains comme Pékin, Wuhan, Xi'an ou Nankin (Amar 2013).

2 Au sens bourdieusien, en tant que « système de valeurs implicites que les gens ont intériorisées depuis l'enfance et à partir duquel ils engendrent des réponses à des problèmes extrêmement différents » (Bourdieu 1984 : 228). 
Les punks de Wuhan, qui sont étudiés dans cet article, se distinguent par le caractère politique de leur musique et les origines sociales de ses membres. La communauté punk de Pékin est en effet considérée - parfois à tort - comme essentiellement issue de la petite-bourgeoisie, certains de ses membres venant de familles relativement aisées ou socialement intégrées, comme les militaires ${ }^{3}$. A l'inverse, les punks de Wuhan sont pour la plupart issus des classes populaires de la ville, et de nombreux punks viennent des villages environnant de la province du Hubei. Lorsqu'ils ont un travail, les punks wuhanais trouvent souvent des emplois à la limite de la légalité et gravitant autour de la scène punk - tatoueurs, serveurs, patrons de bar, dealers, revendeurs d'alcool en gros. Si les punks de Wuhan arrivent à trouver ces emplois, c'est avant tout grâce à leur inscription au sein plus largement des communautés marginales de la société chinoise. Des communautés marginales que l'on a l'habitude de nommer sous le terme de jianghu, qu'il faut replacer dans le contexte historique de son apparition. La notion de jianghu a en effet été popularisée par le maître taoïste Zhuang Zi (IVe siècle avant J.C.). Dans la sixième partie des « chapitres intérieurs » (内篇 - neipian) du texte vraisemblablement apocryphe Zhuang $Z i$, Confucius répond à un disciple venu apprendre auprès de lui les principes du non-agir :

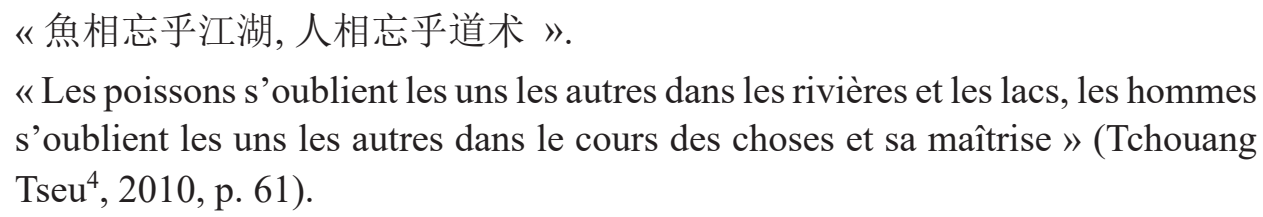

« Les rivières et les lacs », traduction généralement admise pour retranscrire le «jianghu » dans lequel les poissons semblent s'oublier les uns les autres, ne désignent pas qu'un espace physique - l'habitat naturel des poissons. Le jianghu représente selon Zhuang Zi l'état d'insouciance (逍遥), nécessaire à la contemplation du dao (道), de la Voie. L'utilisation du terme jianghu par des poètes comme Du Fu (712-770) lui confère d'autres significations, comme l'émotion ou le sentiment de liberté, qui ont rendu le mot de jianghu extrêmement compliqué à traduire (Yuen Wai 2012). Mais sa signification la plus couramment usitée va coïncider avec la popularisation des romans d'aventure, à l'instar d'Au bord de l'eau, qui font du jianghu une véritable communauté de déviants sociaux au sens de Goffman, c'est-à-dire des individus qui revendiquent collectivement leurs stigmates (Goffman 1975 : 167)

Compilation d'histoires orales narrant les aventures supposées réelles d'une bande d'insurgés de la province du Shandong du XII ${ }^{\mathrm{e}}$ siècle, Au bord de l'eau aurait été composé par Shi Nai' an au XIV siècle. Le bandit Song Jiang, à la tête d'une bande de hors-la-loi, s'insurge contre le gouvernement corrompu des Song du Nord. Le jianghu y désigne alors, dans le contexte du roman de Shi Nai'an, l'espace physique et social où vivent les héros et les brigands, espace doté de ses propres règles et échappant au contrôle des autorités. Dans les romans d'aventure, le jianghu désigne cet espace mythique où les aventuriers, brigands, hors-la-loi et autres escrocs se retrouvent, partageant une même

3 Il faut cependant remarquer qu'aujourd'hui Pékin accueille de nombreux punks venus de province, et la communauté punk de Tongzhou (banlieue est de Pékin) est réputée plus pauvre et plus politique que celle du centre de la capitale.

4 Graphie alternative de Zhuang Zi selon la romanisation de l'Ecole française d'Extrême Orient. 
"Drunk is beautiful ". Boire "au bord de l'eau": usages de l'alcool dans la communauté punk chinoise

défiance envers les autorités et la loi. Mais ce jianghu n'est pas réservé aux œuvres littéraires, il a été constamment réactualisé au sein même de la société chinoise afin de qualifier ceux qui sont mis, ou qui se mettent d'eux-mêmes, hors-la-loi :

«Tandis que le jianghu fait partie de l'imaginaire collectif, il décrit également un espace social réel. C'est le monde marginal des vagabonds, des hors-la-loi, des artistes de l'arnaque, des voleurs, des gardes du corps, des prêteurs sur gage et des collecteurs de dettes, des clochards, des joueurs, des prostituées, et de tous ceux qui vivent seulement par l'épée ou l'intelligence. C'est une hiérarchie très fluide et compétitive, une confrérie indisciplinée dont les membres s'adressent l'un à l'autre comme "grand frère" et "petit frère" " (Boretz 2011 : 12).

Le jianghu représente à la fois l'espace des activités illicites, mais également celui qui permet d'échapper au contrôle social. Dans une société particulièrement hiérarchisée et verrouillée, le jianghu symbolise le « royaume de la liberté où les lois de la famille, de la société et de l'État ne s'appliquent plus »(Shahar 2001 : 380). A ce titre, le jianghu est, aujourd'hui encore, un élément populaire de la culture chinoise, comme en témoigne le succès des wuxia pian, films d'art martiaux qui prennent place au sein de la communauté du jiangh $u^{5}$, ou encore les films sur la mafia, qui revendiquent clairement l'héritage du jianghu ${ }^{6}$. Les productions du cinéma indépendant chinois font elles aussi référence au jianghu, par exemple dans les films de Wang Chao, qui se déroulent pour la plupart dans les bas-fonds mafieux de la société chinoise, à l'instar de L'orphelin d'Anyang (2001) ou Voiture de luxe (2006), où il n'hésite pas à faire appel à de vrais gangsters (Klinger et Privett 2002). Jia Zhangke lui-même invoque le jianghu dans son film $A$ Touch of Sin (2013), dont le titre fait référence à un wuxia pian de King $\mathrm{Hu}, A$ Touch of Zen (1970) ${ }^{7}$.

Par son histoire et sa volonté de créer une communauté en dehors du contrôle des autorités officielles, le punk chinois se rapproche des communautés du jianghu et des " sociétés obscures ». Cette adéquation entre punks et jianghu est particulièrement revendiquée lorsqu'il s'agit de définir la relation des punks aux forces de l'ordre, mais également dans les textes des chansons punks ${ }^{8}$, ou lors des entretiens. Qin Ming* ${ }^{* 9}$, un membre de la communauté punk de Wuhan, analyse ainsi la relation des punks à la police :

«Les flics ne viennent pas nous emmerder. C'est parce qu'on est tous de la mafia (hei shehui), on a peur des flics, et les flics ont peur de nous. Mais attention, on n'est pas des mafieux comme ceux qui font du racket ou qui tabassent des gens. On est en dehors de la société ».

5 Le réalisateur hongkongais Chang Cheh a par ailleurs réalisé en 1972 une adaptation du roman Au bord de l'eau, traduit en français La légende du lac, produit par la Shaw Brothers.

6 On ne compte plus les films hongkongais ou chinois qui font directement référence au jianghu, à l'instar de La voie du jianghu, réalisé en 2004 par Wong Ching-po, dont le titre chinois est simplement Jianghu (江湖).

7 «Maintenant il y beaucoup de choses qui sont semblables à Au bord de l'eau, ou aux films de Chang Cheh et King $\mathrm{Hu}$, tous racontent les choix que font les individus après avoir été confrontés à une pression immense dans un moment de crise ; ils utilisent la violence pour lutter contre la violence, tout cela finissant dans la tragédie » (Jia $2014: 159)$.

8 Citons «江湖大逃亡 », «L'échappée du Jianghu », une chanson de SMZB figurant dans l'album Sin Harmony (2011).

9 Les noms propres suivis d'un * ont été anonymisés. 
Comme nous l'avons précédemment évoqué, la relation des punks au jianghu est structurelle : afin de trouver des lieux où répéter et organiser des concerts, des liens d'amitié et de respect se sont noués entre certains punks et des « grands frères 》 (dage - 大哥) des mafias locales. Ces relations interpersonnelles ne sont pas liées aux règles sociales habituelles, mais se caractérisent selon le « code de l'honneur» (义气), qui implique loyauté et réciprocité dans les relations entre « frères » du jianghu (Boretz 2011 : 35). Le respect dû aux aînés, mais également un sens de l'honneur propre aux 《 frères de la voie obscure » (黑道兄弟) dans le jianghu, est mis en évidence par une anecdote rapportée par Wu Wei :

« Je suis très ami avec le "grand frère" [大哥] ouïghour de Wuhan, il m'appelle “frère” [兄弟 - xiongdi]. Tous les autres ouïghours m'appellent laoda [老大] ${ }^{10}$, dès qu'ils me voient, ils me disent "laoda comment vas-tu ?". La dernière fois, quelqu'un s'est fait voler son portable dans le bar, j'ai appelé le "grand frère", et le lendemain quelqu'un nous l'a rapporté ».

Le mouvement punk, comme toutes les autres communautés du jianghu, fonctionne selon des codes et des hiérarchies spécifiques, même si celles-ci entrent parfois en contradiction avec l'ethos traditionnellement égalitaire du punk occidental, tel qu'il est défini dans les tentatives de conceptualisation d'une " philosophie " punk (O'Hara 2004) qui se veut anti-autoritaire et féministe. Cette inscription du punk chinois au sein du jianghu implique le respect d'une certaine forme de hiérarchie implicite, fondée sur la qualité morale des individus, plutôt que sur leur position sociale, mais également des rituels précis qui symbolisent leur appartenance au jianghu: la consommation d'alcool et la pratique du tatouage, qui se retrouvent également dans d'autres communautés alternatives chinoises.

\section{«For Friends and Beer »-Usages sociaux de l'alcool}

Contrairement à d'autres sociétés, le tatouage en Chine n'a jamais été traditionnellement associé à un rite de passage, mais à une marque d'infamie qui frappait les condamnés et les frères du jianghu, ainsi que les peuples considérés comme barbares, comme les Mandchous et les Yi (Lei 2009 : 102). La prohibition du tatouage se trouvait ancrée dans la tradition confucéenne de la piété filiale, qui interdit aux individus de dégrader le corps reçu de ses parents (Reed 2000 : 364). La communauté du jianghu médiéval se reconnaissait traditionnellement à sa pratique du tatouage, élément déjà présent dans $A u$ bord de l'eau: le tatouage n'était pas associé qu'aux «barbares », mais également aux condamnés qui étaient mis à l'écart de la société par ce tatouage punitif (Reed 2000 : 361) et n'avaient d'autres choix que de se réunir entre déviants au sein du jianghu. Si la pratique du tatouage s'est depuis relativement démocratisée en Chine, elle reste un stigmate eu égard au respect de la piété filiale et reste interdite dans certains corps de métiers officiels. Ainsi, le tatouage peut se révéler une stratégie aux yeux des punks : Pigu, le batteur de SMZB et de The Last Choice (Changsha) était en conflit avec son père qui voulait le voir entrer dans l'armée. Pigu a donc choisi de se tatouer pour éviter la conscription, un homme tatoué ne pouvant faire carrière dans l'armée, et a pu ainsi continuer sa carrière de batteur. La communauté punk chinoise actualise cette 
appartenance au jianghu en s'adonnant collectivement au tatouage comme un rite de passage anti-social, un stigmate assumé ornant le corps punk. Au sens de Goffman, le tatouage des punks représente le stigmate des déviants sociaux qui affichent ainsi leur « refus collectif de l'ordre social. Ce sont eux qui semblent dédaigner les occasions de progresser dans les allées que leur ouvre la société ; eux qui manquent ouvertement de respect à leurs supérieurs ; eux les impies ; eux les échecs de la société quant aux motivations qu'elle propose » (Goffman 1975 : 167). Ce n'est pas un hasard si le bar punk de Wuhan, le Wuhan Prison, a ouvert un magasin de tatouage, le "Wuhan Prison Ink », où une bière est par ailleurs offerte à chaque personne venant s'y faire tatouer. La proximité physique entre le bar et le magasin de tatouage révèle un lien plus fondamental entre la pratique du tatouage et la boisson : elles sont toutes deux des éléments consubstantiels du jianghu, l'une comme stigmate, l'autre comme instrument principal de sociabilité. En outre, pour fonctionner, le tatouage doit posséder plusieurs qualités : il doit être visible, et non pas masqué derrière des vêtements ${ }^{11}$, et doit porter un message compatible avec la démarche punk. Par exemple, la toile d'araignée tatouée sur le coude renvoi plutôt à un engagement skinhead, certains punks portent le tatouage d'un drapeau noir ou un «A » anarchiste comme symbole libertaire, des pistolets ou des couteaux pour marquer leur virilité, ou encore les symboles des groupes de punks euxmêmes. Les punks de Wuhan se tatouent régulièrement «Wuhan Prison » ou encore le logo du groupe SMZB. Pour affirmer ma propre appartenance au punk wuhanais, j'ai tatoué sur mon corps le visuel d'un album de SMZB, ce qui a donné aux punks de Wuhan l'occasion de m'inviter à boire après la finalisation du tatouage en décembre 2014.

Analysant les communautés du jianghu contemporain à travers les écoles d'arts martiaux à Taiwan, Avron Boretz identifie dans les éléments de la fête propre au jianghu la consommation d'alcool comme ferment de la camaraderie. L'importance de l'alcool et son partage sont répandus dans la société chinoise dans son ensemble :
« Les hommes qui boivent ensemble en Chine et à Taïwan apportent sur la table un ensemble spécifique d'attentes et de comportements. La joie apportée par la boisson inclut le plaisir de l'ivresse et l'expérience de la camaraderie. Cependant, l'attente principale n'est pas une sensation physique plaisante, mais bien plutôt l'épreuve de l'appétit, de la capacité et du talent social » (Boretz 2011 : 199).

A l'instar des analyses de Boretz sur le jianghu, la communauté punk chinoise est structurée autour de l'alcool, que cela soit durant les concerts ou dans les bars, espaces privilégiés des réunions punks. L'importance de l'alcool est soulignée par les groupes punks lors des concerts. La qualité de l'accueil et de l'organisation est mesurée par la quantité et la qualité de l'alcool mises à la disposition des musiciens. Les groupes qui jouent se voient d'ordinaire octroyer gracieusement de l'alcool en prime d'une somme d'argent prélevée sur les billets d'entrée. Parfois, il arrive que les gérants des salles de concert fassent preuve de malhonnêteté, comme s'en souvient Zhao Yu, le chanteur du groupe pékinois Discord :

« La dernière fois qu'on a joué au Mao Livehouse [à Pékin], le patron s'est

11 Ainsi lors d'un entretien, un punk de Wuhan en critiquait un autre, qui s'était fait uniquement tatouer sur le torse : « on ne le voit pas, ce n'est pas un tatouage punk! », entretien avec Yang Han et Zuo Yan, Pékin, 10 avril 2015. 
vraiment mal comporté. Quand on est arrivé pour jouer, on a eu le droit qu'à une seule bouteille de Tsingtao ${ }^{12}$ par personne. Une seule! Ensuite, au moment de nous payer, il était déjà parti avec la caisse. On a dû le menacer au téléphone pour qu'il revienne nous payer. Ça me rappelle la fois où on est allé à Nankin pour jouer dans un bar. Le patron nous offre à boire, il nous dit "buvez autant que vous voulez". Au moment de nous payer, il nous dit : "Voilà ce que je vous dois, moins l'alcool que vous avez bu”. Quel enfoiré [俊逼]!».

La performance musicale punk implique un don d'alcool de la part des bars où se produisent les groupes. Cela marque le respect dû aux groupes, qui se révèle souvent plus important aux yeux des musiciens que le maigre cachet qui accompagne leur concert. Lorsque l'alcool est servi abondamment, le concert est considéré comme réussi. $\mathrm{Wu}$ Wei, le chanteur de $\mathrm{SMZB}$, met ainsi en parallèle deux concerts en fonction de l'alcool qui a été mis à la disposition des musiciens :

"Quand on est allé au Mao de Shanghai, le patron nous a donné six bières. Six bières ! Pas six bières par personnes, six bières en tout ! Ici [à Wuhan], quand on joue, le Vox ${ }^{13}$ nous offre une bouteille de whisky, c'est comme notre maison! ».

L'économie punk est avant tout fondée sur le don et le contre-don d'alcool, à l'instar de la pratique du don opérée dans la société chinoise, qui articule l'émotion (ganjing) et l'utilité (Kipnis 1996 : 288). Pendant un concert, l'alcool se doit d'être offert au groupe, mais aussi à son entourage proche, comme l'ingénieur du son ou le roadie ${ }^{14}$. Les hiérarchies se manifestent dans les rituels alcooliques : qui commande l'alcool, qui paye, qui commence à trinquer, tous ces gestes sont conditionnés par des normes implicites. Ainsi, lorsqu'un groupe est invité à tourner, les organisateurs l'invite à manger et paye le repas et la boisson ; on laisse le chanteur commander son alcool de prédilection qui doit être ensuite partagé par toute la tablée. Ayant moi-même participé à une tournée de $\mathrm{SMZB}$, j'ai pu prendre part aux préparatifs du concert du groupe à Qingdao. Pendant les répétitions, le bar qui accueillait le concert, le Downtown, avait mis à la disposition du groupe de nombreuses bières que les membres de SMZB se sont partagées. Durant le concert, alors que je tentais d'acheter une bouteille de bière au bar, on m'a clairement signifié que je ne devais pas payer :

«Tu fais partie du groupe, tu ne dois pas payer. C'est comme ça que l'on fait avec les frères [哥们 - gemen] de Wuhan ».

Ce potlatch éthylique n'est pas réservé aux rapports entre les seuls membres des groupes de punk ni à l'espace des salles de concert, mais irrigue les rapports entre les punks eux-mêmes. Ces échanges réciproques ne sont pas circonscrits à l'alcool et concerne également la cigarette et - dans une moindre mesure - la marijuana. Les punks s'offrent mutuellement de l'alcool, partagent leurs bouteilles, et participent régulièrement à des concours de jeux à boire. Pour décider de façon non autoritaire de l'ordre de passage des groupes de punk lors des différentes éditions du Strawberry Fucktival (le contre-festival punk organisé tous les ans à Tongzhou, une banlieue est

12 Bière chinoise populaire produite dans la ville portuaire de Qingdao

13 Salle de concert alternative la plus importante de Wuhan.

14 Un roadie, ou un machiniste itinérant, accompagne les groupes de musique lors de leurs tournées. Dans le cas des groupes de punk, ce sont souvent des amis du groupe, et ils intègrent souvent à terme le groupe en tant que musiciens. 
de Pékin depuis mai 2013) ${ }^{15}$, les groupes ont mis en place une technique originale : les groupes qui vident leur pinte de bière le plus rapidement peuvent choisir en premier leur ordre de passage.

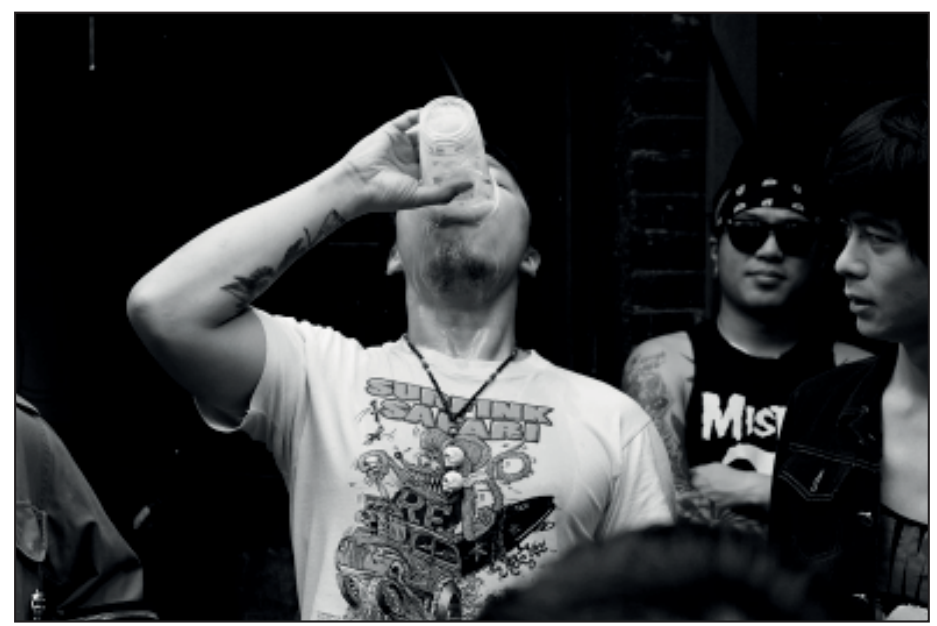

Figure 1 : Le bassiste de The Diders vide sa pinte lors du concours de bière au DMC, Tongzhou, $1^{\text {er }}$ mai 2013 - (c) Nathanel Amar, 2013

Dans le cas du concours de bière organisé lors du festival de punk de Tongzhou, l'alcool représente bien plus qu'un instrument d'échange : il fait partie d'un modèle alternatif d'organisation. L'ordre de passage des groupes lors d'un concert est un enjeu important : ceux qui passent en premier ou en dernier risquent de jouer devant une salle vide. Cet ordre est d'habitude décidé au préalable de manière obscure par les organisateurs suivant leur préférence ou la notoriété du groupe. Cette pratique est très contestée chez les punks chinois, qui la trouvent très peu égalitaire. Pour rendre le processus plus démocratique, les punks de Tongzhou ont donc eu l'idée d'organiser ce concours de bière. Bien entendu, certains groupes sont désavantagés par leurs membres moins portés sur l'alcool, comme l'a confié le chanteur de Discord Zhao Yu : « on a dû passer en dernier, c'est à cause du guitariste, il boit trop lentement ! ». L'alcool impose une nouvelle hiérarchie construite sur la capacité à ingurgiter le plus rapidement possible une pinte de bière, et qui constitue la « valeur » de l'individu.

L'alcool tient une place particulière dans l'imaginaire punk et surtout la bière, peu chère et aisément accessible. A ce propos, de nombreuses chansons de punk font l'éloge de l'ivresse ou de l'alcool. Joyside, groupe punk-rock pékinois majeur du milieu des années 2000, publie deux albums aux titres évocateurs, Drunk Is Beautiful en 2005 et Booze At Neptune's Dawn en 2007. On y retrouve des chansons comme «I Want Beer», « Fall In Beer With You », ou encore « Booze At Dawn ». Ce groupe et ses proches

15 Tous les ans, le label de rock « indépendant» (mais appartenant à une maison de disque officielle) Modern Sky organise un festival dans la banlieue de Tongzhou, le « Strawberry Festival ». Les organisateurs de l'événement refusent d'accueillir les punks, qui peuplent pourtant Tongzhou. Ces derniers ont donc décidé d'organiser leur propre festival, le « Strawberry Fucktival», sorte de pied de nez au festival officiel. 
créeront le groupe informel «Gang of Gin » ${ }^{16}$, à l'origine de la création du bar punk « School Bar » à Pékin. Chez Joyside, la bière est considérée comme une échappatoire à un quotidien morne, ainsi qu'en témoigne la chanson «I Want Beer » :

What a dirty goddam world

Too much shit I cannot stand

Everybody's a nightmare

My sweet home's just a hell

So fuck it!!!

I want beer, I want beer, I want beer, I want beer

Oh dear mankind god damn you

I do have a heart but it's not for you

Now shut up your stupid faces

I never care about you bastards

Fuck you!!!

I want beer, I want beer, I want beer, I want beer

Oh what the fuck is this?

I can't bare it anymore

What I need's

Just more more more more more beer

I want beer, I want beer, I want beer, I want beer

I really want, please give me some more ${ }^{17}$

De nombreuses chansons rapprochent ainsi la consommation d'alcool à une contestation de l'ordre social existant. Si Joyside prétend sombrer dans l'alcool pour échapper à la dépression, le groupe de Changsha The Last Choice revendique dans sa chanson «I Don't Care » la consommation de bière comme mode de vie, en opposition à la société policée des « costards-cravates » :

城市街上住着一群混蛋（那是我们, 就是我们)

Dans les rues de la ville vit une bande de bâtards (ça c'est nous, c'est bien nous)

拿着酒瓶四处制造混乱（就要给你一点颜色看看）

On tient des bouteilles d'alcool et on fout le bordel partout (ça c'est pour te

donner une bonne leçon)

西装领带都骂我们坏蛋

Les costards-cravates nous traitent de branleurs

I don't care I wanna beer

I don't care I wanna fucking beer

I don't care I need fucking more

I don't care I drink anywhere ${ }^{18}$

En cela, la fonction de l'alcool se rapproche du stigmate volontaire que constitue le tatouage en se faisant symbole de la séparation qu'effectuent les punks avec la

16 Référence à une chanson du groupe anglais Babyshambles, dont le chanteur, Pete Doherty, ne cache pas son penchant pour la boisson.

17 Joyside. «I Want Beer », in Drunk Is Beautiful. Modern Sky, 2005. La chanson est interprétée en anglais.

18 The Last Choice. «I Don’t Care » in Fuck War, Autoproduit, 2007. Le refrain est chanté en anglais. 
"Drunk is beautiful ". Boire "au bord de l'eau": usages de l'alcool dans la communauté punk chinoise

société. La chanson de The Last Choice indique bien que la consommation d'alcool n'est pas qu'une affaire privée, elle s'exprime dans la rue, de manière publique, où les bandes de punks « foutent le bordel partout». Cette consommation s'oppose en ce sens à celle des cadres du Parti ou des cols blancs, qui s'enivrent lors de banquets onéreux pour entériner des accords commerciaux ou politiques, tout en restant dans le cadre privé des restaurants ou des bars de karaoké. L'alcool permet pour les punks d'agir dans la rue, la bouteille elle-même pouvant servir d'arme, ou lorsqu'ils courent nus dans la rue sous l'emprise de l'alcool. L'ivresse publique contrevient en effet aux politiques de 《 civilisation 》 (文明 - wenming) de la société chinoise, initiée depuis les réformes de Deng Xiaoping entamées au début des années 1980 (Dynon 2008 : 86). Ces injonctions à la « civilité » se déclinent aussi bien sur les murs des villes par le Bureau de construction de la civilisation spirituelle (Kloeckner 2016) qu'au niveau des quartiers d'habitation où les comités de résidents diffusent des normes de savoirvivre et de bienséance aux habitants (Audin 2015 : 101). Les comportements «non civilisés », comme fumer dans les restaurants, cracher dans la rue ou l'ivresse publique, font régulièrement l'objet de campagnes de propagande. En ce sens, l'éloge de l'alcool par les punks s'oppose à cette « civilisation » perçue comme l'un des mots d'ordre du Parti communiste. Nombreux sont les punks arrêtés par la police pour ivresse sur la voie publique, à l'instar du chanteur et du bassiste du groupe pékinois Discord qui ont passé une semaine en prison pour s'être battus alors qu'ils étaient ivres. Marque de respect, d'amitié et stigmate volontaire, l'alcool fait partie intégrante de la vie punk. Se pose néanmoins la question pour l'ethnographe de son rapport à l'alcool dans un tel contexte : si la consommation d'alcool est indispensable à l'entrée sur le terrain, comment aborder l'enquête en état d'ébriété ? L'alcool pose-t-il un problème dans la conduite des entretiens et pour la qualité des observations ?

\section{One of the boys - Faire de la recherche en état d'ébriété}

Faire une recherche sur la communauté punk, comme dans toute autre communauté déviante, requiert du temps et une immersion totale si l'on veut donner à ses descriptions et entretiens un sens autre que superficiel. La recherche que j'ai menée de 2012 à 2015 au sein des communautés punks chinoises s'est donc déployée dans un temps long, nécessaire à mon intégration au sein des punks. La tradition sociologique fournit un nombre important de paradigmes ethnographiques permettant de théoriser le rapport du chercheur à son sujet. Les analyses séminales de William Foote Whyte sur un coin de rue à Chicago ont permis de développer l'observation participante, seule à même de rendre compte des interactions quotidiennes et à en relever les enjeux ${ }^{19}$. Mais c'est sans doute le travail ethnographique de Philippe Bourgois dans In search of respect, sur des dealers de crack dans le quartier d'El Barrio à Harlem, qui est le plus à même d'appréhender le travail de terrain que j'ai effectué auprès des punks chinois. Philippe Bourgois n'a pu mener son enquête qu'en construisant une relation de confiance durable avec les dealers, étant lui-même un habitant du quartier d'El Barrio. C'est la méthode ethnographique de l'observation participante qui lui a permis de réaliser son travail,

19 « Si l'on veut comprendre un individu, il faut le replacer dans son environnement social et l'observer dans ses activités quotidiennes [...] un membre de la classe moyenne ne verra dans les quartiers pauvres qu'une confusion extrême, qu'un chaos social. Pour celui qui y vit, Cornerville apparait comme un système social très fortement organisé et intégré » (Whyte $2007: 36$ ). 
car c'est selon lui :

« seulement en établissant des relations sur le long terme fondées sur la confiance que l'on peut commencer à poser des questions personnelles et provocantes, et s'attendre à des réponses réfléchies et sérieuses [...] En d'autres mots, afin de collecter des "données précises", les ethnographes violent les canons de la recherche positiviste ; nous devenons intimement impliqués avec les personnes que nous étudions » (Bourgois $2010: 13$ ).

Cette implication peut aussi se transformer en adéquation, comme le souligne Wacquant, qui a réussi à surmonter sa position initiale d'outsider pour son ethnographie d'un club de boxe à Chicago, en devenant " one of the boys" (Wacquant 2002 : 8). Réussir à intégrer le club n'était pourtant pas une évidence, Loïc Wacquant étant le seul blanc et étranger de la salle de boxe. Il lui a donc fallu « mettre les gants » (Wacquant 2002 : 7) et participer aux activités des boxeurs, jusqu'à devenir l'un d'entre eux. Devenir « one of the boys » dans le contexte du punk chinois implique de participer aux activités collectives des punks, et donc de boire (souvent beaucoup) d'alcool, cette pratique étant, comme nous l'avons vu, une composante essentielle de la sociabilité punk. L'engagement corporel du chercheur sur son terrain est un fait déjà bien analysé dans la tradition ethnologique, elle permet de comprendre les relations sociales qui se tissent lors des jeux de boisson, et qui demandent la participation active du chercheur (Fiskesjö 2010). Cette participation corporelle ne se limite pas à l'alcool, elle englobe toutes les pratiques rituelles comme le partage du repas, dont il faut assimiler les codes afin de ne pas vexer ses enquêtés, tout en sachant apprendre à refuser pour préserver sa santé (Kradolfer 2006). Il est cependant difficile de refuser « le dernier verre » lorsqu'il vient sceller l'amitié mais empêche de conduire son enquête jusqu'au bout : ainsi, en consultant mon carnet de terrain, le 15 décembre 2012, j'ai écrit qu'il m'était impossible de continuer à prendre des notes à cause de mon état d'ébriété. C'est à partir de ce moment que j'ai commencé un apprentissage de mes limites et de différentes techniques pour continuer à boire tout en poursuivant l'enquête ethnographique.

Ce n'est pas, bien entendu, par ma seule consommation d'alcool que j'ai pu devenir « one of the boys », d'autres facteurs - dont la pratique de la langue chinoise, la connaissance de l'histoire (contre-)culturelle chinoise, des référents culturels et politiques communs - peuvent expliquer mon intégration au punk chinois. Mais c'est en montrant en quelque sorte ma « valeur » dans le domaine de l'alcool, une pratique dérivée du jianghu (Boretz 2011 : 185), que j'ai acquis un statut d'insider - à savoir montrer ma résistance à l'alcool, offrir de l'alcool et en recevoir, trinquer, participer aux jeux à boire. Mettre en jeu son corps au sein de sa recherche est une pratique courante dans l'ethnographie. Outre Wacquant, Pierig Humeau, dans son étude de l'espace punk français, a conduit une « observation totale où [s]on corps [ét]ait positionné comme outil de recueil de données » (Humeau et Rachamim 2009 : 51). L'apprentissage par l'hexis corporelle du mode de vie punk - extériorisation de l'habitus à travers par exemple le style vestimentaire ou la technique musicale - permet d'acquérir une position d'insider. N'étant que très peu doué musicalement, à la différence de Pierig Humeau, mon apprentissage corporel s'est centré sur l'adoption des codes vestimentaires, du stigmate visible (tatouage) et du partage de l'alcool. Une fois accepté en tant que compagnon de boisson, mon statut a changé, ce qui m'a été signifié par un jeu de mot : je n'étais plus un 《 étudiant en recherche » «yanjiu sheng 》 (研究生), mais un 《étudiant en alcool et 
clope », «yan jiu sheng » (烟酒生) ${ }^{20}$. Cette intégration s'est confirmée lorsqu'un punk de Wuhan, Yang Han, m'a présenté à d'autres amis punks non comme un étranger ou un chercheur, mais comme un membre de la communauté punk de Wuhan :

«Lui c'est Tairan [le nom de l'auteur en chinois]. Ce n'est pas un étranger, c'est un Wuhanais. L'un des nôtres ».

Cette intégration par l'alcool peut poser des interrogations d'ordre pratique : comment conduire une recherche en état d'ébriété ? L'alcool peut en effet altérer les sens du chercheur, l'empêcher de conduire des entretiens et dégrader la qualité de ses observations. Cette critique suppose qu'il existe un « état normal », différent de l' « état d'ébriété », qu'il serait préférable d'adopter lors de la recherche. Il me semble plutôt que conduire une recherche ethnographique de qualité demande au chercheur de partager avec le sujet de ses recherches le même « état ». Ma conviction est qu'il n'y a pas d' " état » normal ou optimal pour réaliser sa recherche : être sous l'emprise de l'alcool, lorsque ces substances sont partagées par le groupe que l'on étudie, permet d'expérimenter les interactions telles qu'elles sont vécues par les participants euxmêmes. Ainsi, il faut par exemple avoir passé sa soirée à boire avec des punks pour comprendre la pratique courante du luoben (裸奔), comme l'évoque Wu Wei :

« Notre sport favori à Wuhan, c'est le luoben. Quand il est tard et que tu es saoul, tu enlèves tes vêtements et tu cours tout nu dans la rue. Souvent la police nous court après ».

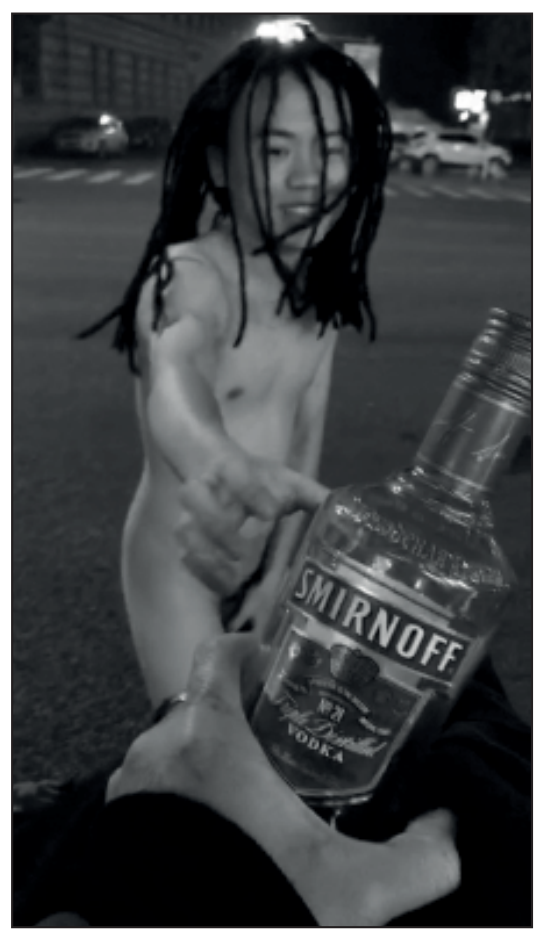

Figure 2 : un punk pratique le luoben à Wuhan, décembre 2016 - (C) Nathanel Amar, 2016

20 Jeu de mots difficilement traduisible, qui joue sur l'homophonie entre « recherche » (yanjiu) et « alcool et cigarette » (yan jiu). 
Le luoben n'est pas qu'un jeu puéril, c'est un rite de passage, une preuve de son courage, à la fois de courir nu dans la rue et de défier la police, mais aussi l'expression de sa liberté. Les punks de Wuhan se nomment par ailleurs eux-mêmes les «裸体朋 克》, les 《Naked Punks ». La consommation d'alcool est également liée à un moment de confession, où l'on parle plus facilement de soi et d'expression politique éruptive. Comme l'exprimait Cai Qing* à propos d'un client du bar, « lui, quand il a trop bu, il a l'habitude de baisser son pantalon et de montrer son sexe en criant "Fuck Wen Jiabao" 21 " [ancien premier ministre chinois] ». La profusion de la parole permise grâce à l'alcool peut également se manifester durant les concerts, lorsque le chanteur a trop bu. Lors du concert de SMZB du 21 décembre 2012, le chanteur Wu Wei a longuement harangué la foule, et a tenu un long discours sur le rôle du chanteur de punk face au gouvernement chinois. Si la prise de parole de Wu Wei durant ses concerts est systématique, la longueur de celle-ci varie en fonction de son taux d'alcoolémie, ainsi qu'il me l'avouera le lendemain :

« On m'a dit que j'ai trop parlé hier. C'est parce que j'avais beaucoup bu avant de monter sur scène. A chaque fois que je bois trop, je parle trop. Mais tu sais pourquoi je le fais? Parce qu'en Chine on a trop peu l'occasion de parler, de dire ce que l'on pense. Alors dès que j'en ai l'occasion, je parle ! ».

Il existe en chinois une expression similaire à in vino veritas, 《酒后吐真言 》 («jiuhou tu zhenyan »), littéralement « après l'alcool, vomir la vérité ». Il serait tentant de dire que la consommation d'alcool permet de vomir également la politique, comme en témoignent les derniers exemples. Les punks n'ont cependant pas besoin d'alcool pour critiquer le gouvernement et l'insulter - ce qu'ils font régulièrement à jeun -, mais l'intoxication rend la critique plus agressive. Rapporter ces paroles avinées, dans leur contexte d'énonciation, a été essentiel dans la recherche que j'ai menée, mais pour ce faire il a fallu abolir les frontières symboliques qui existent entre le chercheur et son sujet de recherche. Ce sont aussi des « émotions » (ganjing) qui s'expriment lors de la consommation d'alcool, alors que l'expression des émotions est le plus souvent réprimée dans la société chinoise. Si la parole peut devenir politique une fois l'alcool bu, elle est aussi " émotionnelle », au sens où les langues se délient et que le fait de trinquer renforce les liens entre deux ou plusieurs individus, comme le remarque Andrew Kipnis, « le fait de trinquer matérialise le respect, tandis que le fait de boire déconstruit les frontières séparant [...] hôtes et invités, permettant ainsi la circulation des sentiments » (Kipnis 1997 : 54). C'est après avoir beaucoup bu que mes interlocuteurs punks ont abordé les sujets les plus sensibles, qu'ils n'expriment pas généralement : soucis sentimentaux, existentiels ou financiers ${ }^{22}$. Si les rituels qui précédent l'ingestion d'alcool marquent la hiérarchie, la consommation permet de rétablir une égalité entre les participants, engendrant une retenue moins grande dans l'expression des sentiments et la diffusion de la parole. L'alcool, et la réciprocité que permet sa consommation, permet de mettre sur un même pied d'égalité le punk et le chercheur, jusqu'à ce que celui-ci devienne " one of the boys », ou plutôt, pour reprendre une chanson du groupe pékinois The Diders, un « beer boy».

21 En anglais.

22 Je ne m'étendrai pas plus avant sur ces confidences, qui n'ont pas été faites au chercheur, mais à l'ami. 
"Drunk is beautiful ". Boire "au bord de l'eau": usages de l'alcool dans la communauté punk chinoise

\section{Conclusion}

En me rendant un soir au bar, je croise Yang Han affalé sur le comptoir. Je lui demande s'il veut que je lui offre quelque chose à boire, à quoi il me répond : " prends-moi un huihun jiu (回魂酒) ». Je n'avais jamais entendu parler de cet alcool, et lui demande si c'est une bière : « non, me dit-il, c'est quand tu as trop bu la veille, tu prends n'importe quel autre alcool pour te réveiller ». Littéralement " alcool qui rend l'âme », le huihun jiu désigne tout alcool destiné à se remettre d'une soirée arrosée. L'alcool rythme littéralement le quotidien d'un punk, de la bière partagée lors du repas, à la bouteille de whisky pour se préparer à un concert, jusqu'à la pinte renversée lors d'un pogo, puis à l'alcool du lendemain qui symbolise le retour de l'âme. Loin d'être accessoire, l'alcool est avant tout un instrument social qui se doit d'être partagé, offert, reçu, selon des codes précis, hérités des communautés du jianghu. La particularité de l'alcool chez les punks chinois, outre d'être lié à des rituels sociaux, est de faire partie intégrante du discours et des chansons punks. La consommation d'alcool est liée, dans ces textes, à une contestation de l'ordre social existant contre les efforts entrepris par le Parti communiste pour « civiliser » (文明) la population chinoise ; les punks revendiquent fièrement leur volonté de ne pas s'y conformer. Si nous avons pu relever autant de significations différentes liées à l'alcool, c'est avant tout en consommant de l'alcool avec les punks, et ainsi pouvoir atteindre un état d'égalité entre le chercheur et son sujet. En ce sens, l'alcool permet de « faire bégayer la langue », comme le perçoit Deleuze dans Six fois deux de Godard où ce dernier arrive à « traiter d'égal à égal avec n'importe qui » (Deleuze 1990 : 55). Tout comme il faut mettre des gants lors d'une enquête sur la boxe, il a fallu boire des bières lors de cette recherche sur les punks chinois, jusqu'à donner une consistance alors insoupçonnée à la formule de SMZB, « for friends and beer $»$.

\section{Références citées}

Amar, Nathanel, 2013. «Une histoire politique du punk-rock chinois », Circé. Histoire, culture \& société, en ligne : <http://www.revue-circe.uvsq.fr/une-histoire-politique-du-punk-rock-chinois/> [consulté le 10 janvier 2017].

Audin, Judith, 2015. « Gouverner par la communauté de quartier (shequ) en Chine. Ethnographie de la bureaucratisation participante des comités de résidents à Pékin », Revue française de science politique, 65 (1), pp. 85-110.

Boretz, Avron, 2011. Gods, Ghosts, and Gangsters. Hawaï: University of Hawai's Press.

Bourdieu, Pierre, 1984. Questions de sociologie. Paris : Minuit.

BourgoIs, Philippe, 2010. In search of respect: Selling crack in El Barrio. Cambridge: Cambridge University Press.

De KloEt, Jeroen, 2010. China with a Cut. Globalization, Urban Youth and Popular Music. Amsterdam: Amsterdam University Press.

Deleuze, Gilles, 1990. Pourparlers. 1972-1990. Paris : Minuit

DynOn, Nicholas, 2008. "'Four Civilizations' and the Evolution of Post-Mao Chinese Socialist Ideology”, The China Journal, 60, pp. 83-109.

Goffman, Erving, 1975. Stigmate. Les usages sociaux des handicaps. Paris : Minuit. 
GRILlot, Caroline, 2001. "Dixia Chengdu/Chengdu Underground", Ateliers, 24, <https://ateliers.revues. org/8721> [consulté le 15 janvier 2017].

FisKesJö, Magnus, 2010. "Participant Intoxication and Self-Other Dynamics in the Wa context", The Asia Pacific Journal of Anthropology, 11 (2), pp. 111-127.

Humeau, Pierig et Yechezkel Rachamim, 2009. «Quand le chercheur est 'One of the boys'... », Regards sociologiques, 37-38, pp. 45-66.

JIA, Zhangke (dir.), 2014. Tian zhu ding. Shandong : Shandong chuanmei chubanshe.

KIPNIS, Andrew B., 1996. "The Language of Gifts. Managing Guanxi in a North China Village", Modern China, 22 (3), pp. 285-314.

KIPNIS, Andrew B., 1997. Producing Guanxi: sentiment, self and subculture in a North China Village. Durham: Duke University Press.

Klinger, Gabe et Ray Privett, 2012. "An Independent Chinese Voice: Wang Chao And The Orphan of Anyang", Indiewire <http://www.indiewire.com/article/interview_an_independent_chinese_voice_ wang_chao_and_the_orphan_of_anyang> [consulté le 16 mai 2016]

KLOECKNER, Léo, 2016. « L'image de propagande en Chine, outil du contrôle social : le cas de Pékin », Geoconfluences $<\mathrm{http}$ //geoconfluences.ens-lyon.fr/informations-scientifiques/dossiers-regionaux/lachine/corpus-documentaire/image-de-propagande-en-chine> [consulté le 28 mai 2016].

KRADOLFER, Sabine, 2006. «Boire et manger : l'épreuve du terrain », Journal des anthropologues, 106-107, pp. 297-305.

LeI, Daphne P., 2009. "The Blood-Stained Text in Translation: Tattooing, Bodily Writing, and Performance of Chinese Virtue", Anthropological Quarterly, 82 (1), pp. 99-127.

Link, Perry et Richard P. MADSEN, 2002. Popular China. Unofficial Culture in a Globalizing Society. Oxford: Rowman \& Littlefield.

Lü, Xinyu, 2003. Jilu Zhongguo [Documenter la Chine]. Pékin : Sanlian.

MaI, Dian, 2012. «Yige pengke de jichu linglei jiaoyu » [« Les fondements de l'éducation alternative d'un punk »], Tiannan [Chutzpah!] 6, pp. 102-112.

O'Hara, Craig, 2004. La philosophie du punk. Histoire d'une révolte culturelle. Saint Mury-Monteymond : Rytrut.

Pickowicz, Paul G. et Yingjin Zhang (eds), 2006. From Underground to Independent. Alternative Film Culture in Contemporary China. Oxford: Rowman \& Littlefield.

ReED, Carrie E., 2000. "Tattoo in Early China”, Journal of the American Oriental Society, 120 (3), pp. 360376.

Shahar, Meir, 2001. "Ming Period Evidence of Shaolin Martial Practice", Harvard Journal of Asiatic Studies, 61 (2), pp. 359-413.

TchouAng Tseu (Traduction de Jean LÉvI), 2010. Les æeuvres de maître Tchouang. Paris : Encyclopédie des nuisances.

WACQUANT, Loïc, 2002. Corps et âme. Carnet ethnographique d'un apprenti boxeur. Marseille : Agone.

Whyte, William F., 2007. Street Corner Society : La structure sociale d'un quartier italo-américain. Paris : La Découverte.

YANG, Jian, 2013. 1966-1976 de dixia wenxue [La littérature clandestine de 1966 à 1976]. Pékin : Zhonggongdang shi chubanshi.

YUEN WAI, Helena, 2012. "A Journey across Rivers and Lakes: A Look at the Untranslatable Jianghu in Chinese Culture and Literature", $452 \mathrm{oF}$. Electronic journal of theory of literature and comparative literature, No. 7, pp. 58-71 <http://www.452f.com/pdf/numero07/07_452f-mono-helena-yuen-waiorgnl.pdf $>$ [consulté le 16 mai 2016]. 


\section{Euvres musicales citées}

JoysiDE, 2005. Drunk Is Beautiful. Modern Sky.

Joyside, 2007. Booze At Neptune's Dawn. Maybe Mars.

SMZB, 2007. For Friends and Beer. Autoproduit.

SMZB, 2008. Ten Years Rebellion. Maybe Mars.

SMZB, 2011. Sin Harmony. Maybe Mars.

The Last Choice, 2007. Fuck War. Autoproduit.

THE DiDERS, 2014. EP. D.O.G. 